\title{
Sheath capacitance observed by impedance probes onboard sounding rockets: Its application to ionospheric plasma diagnostics
}

\author{
Tomonori Suzuki ${ }^{1}$, Takayuki Ono ${ }^{1}$, Jyunpei Uemoto ${ }^{2}$, Makoto Wakabayashi ${ }^{3}$, \\ Takumi Abe ${ }^{4}$, Atsushi Kumamoto ${ }^{1}$, and Masahide Iizima ${ }^{5}$ \\ ${ }^{1}$ Graduate School of Science, Tohoku University, Sendai 980-8578, Japan \\ ${ }^{2}$ National Institute of Information and Communications Technology, Tokyo 184-8795, Japan \\ ${ }^{3}$ Niihama National College of Technology, Ehime 792-8580, Japan \\ ${ }^{4}$ Institute of Space and Astronautical Science, Sagamihara, Kanagawa 229-8510, Japan \\ ${ }^{5}$ Shukutoku University, Tokyo 174-8645, Japan
}

(Received August 18, 2009; Revised January 23, 2010; Accepted January 28, 2010; Online published August 31, 2010)

\begin{abstract}
Ion sheath which is formed around an electrode signi cantly affects the impedance of the probe immersed in a plasma. The sheath capacitances obtained from impedance probe measurements were examined for application to plasma diagnoses. We compared analytical calculations of the sheath capacitance with measurements from impedance probes onboard ionospheric sounding rockets. The S-520-23 sounding rocket experiment, which was carried out in mid-latitude, demonstrated that the observed sheath capacitances agreed well with those of the calculations. We concluded that the sheath capacitance measurements allow for estimation of the electron temperature and the electron density of a Maxwellian plasma. On the other hand, the sheath capacitances obtained from the S-310-35 rocket experiment in the auroral ionosphere showed lower values than expected. Auroral particles precipitations should modify the probe potential.
\end{abstract}

Key words: Impedance probe, sheath capacitance, plasma diagnostics, ionosphere.

\section{Introduction}

In-situ plasma diagnoses are essential for clarifying various aspects of phenomena found in space and laboratory plasmas. A radio frequency $(\mathrm{RF})$ probe technique is a powerful tool for measuring the plasma parameters (e.g., Jackson and Kane, 1959). Over the past several decades, numerous investigations have been conducted on the impedance of an electrode immersed in a plasma in order to achieve accurate plasma diagnoses.

An impedance probe, which is a kind of RF plasma probe, has been developed as an unique tool to measure the electron number density. The probe impedance in a magnetized plasma has a resonance at the upper hybrid resonance (UHR) frequency $f_{\mathrm{UHR}}$

$$
f_{\mathrm{UHR}}^{2}=f_{\mathrm{ce}}^{2}+f_{\mathrm{pe}}^{2},
$$

where $f_{\text {ce }}$ and $f_{\mathrm{pe}}$ denote the electron cyclotron frequency and the electron plasma frequency, respectively. Measurement of frequency dependence of the probe impedance, therefore, allows us to derive the electron density from the UHR frequency. To eliminate measurement errors of the electron density due to the stray capacitance in the electric circuit, Oya and Obayashi (1966) designed the impedance probe to measure the impedance curves by using the capacitance bridge. Accordingly, the impedance

Copyright (C) The Society of Geomagnetism and Earth, Planetary and Space Sciences (SGEPSS); The Seismological Society of Japan; The Volcanological Society of Japan; The Geodetic Society of Japan; The Japanese Society for Planetary Sciences; TERRAPUB.

doi:10.5047/eps.2010.01.003 probe made it possible to measure the absolute electron density with a high degree of accuracy. Since then, various types of improved impedance probes have been installed on many sounding rockets (e.g., Yamamoto et al., 1998; Wakabayashi et al., 2005; Barjatya and Swenson, 2006) and scienti c satellites (e.g., Ejiri et al., 1973; Oya and Morioka, 1975; Takahashi et al., 1985; Watanabe and Oya, 1986) for measuring electron density.

The observed antenna impedance re ects various physical properties of the ambient plasma as well as the electron density. Evaluations of the observed impedance are actively discussed subjects these days. Tsutsui et al. (1997) examined antenna impedance measured by the Geotail spacecraft in the Earth's magnetosphere, where the electron densities are too low to observe the resonances. Béghin et al. (2005) computed the self-impedance and mutual-impedance to model the electric antennas onboard the Cluster satellites. Impedance measurements in a laboratory plasma showed resonances of a long dipole antenna (Blackwell et al., 2007). Miyake et al. (2008) developed an analysis tool of antenna impedance via Particle-In-Cell (PIC) simulation. A Plasma-Fluid Finite-Difference Time Domain (PF-FDTD) simulation was applied to estimate the collision frequency in the ionosphere (Ward et al., 2005; Spencer et al., 2008). In addition, unique characteristics of the probe impedance in a thermal magnetized plasma were found by laboratory experiments (Suzuki et al., 2009).

In actual situations, for evaluating the probe impedance it is essential to consider the effect of an ion sheath surrounding the probe. This paper deals with the capacitance of the 
ion sheath measured by impedance probes. The ion sheath is formed around the probe due to the difference between the thermal velocity of electrons and ions. The sheath capacitance signi cantly affects the probe impedance at lower frequencies in comparison to that around the UHR frequency. Derivation of the electron temperature from the sheath capacitance was originally proposed by Oya and Aso (1969). Watanabe (2000) reported that sheath thickness was changed with the auroral energetic electron ux. Sheath capacitance has also been studied in terms of its capacitively coupled plasma (e.g., Chen, 2006).

The purpose of this study is to evaluate the sheath capacitance obtained from impedance probe measurements in order to utilize it for ionospheric plasma diagnostics. We applied an analytical formula of the sheath capacitance with a step sheath model. Although our calculation method of the sheath capacitance is fundamentally the same as that of the report by Oya and Aso (1969), we have made some modi cations on the evaluation of the probe potential. We examined the sheath capacitance observed from impedance probes onboard the two sounding rockets, S-520-23 and S310-35. First, we show analysis of the sheath capacitance in a quiet state of the ionospheric plasma to con rm the validity of the analytical model. We also provide observations of the sheath capacitance under disturbed conditions.

\section{Sheath Capacitance in Impedance Probe Mea- surements}

We applied a simple sheath model as illustrated in Fig. 1. There are no electrons in the sheath region whose radius is $R_{\mathrm{s}}$, while ions are distributed uniformly. The equivalent probe capacitance $C_{\mathrm{pr}}$, which is the output signal from the impedance probe, consists of the sheath capacitance $C_{\mathrm{s}}$ and the equivalent capacitance of the plasma region $C_{\mathrm{pl}}$

$$
\begin{aligned}
C_{\mathrm{pr}} & =\left|\frac{C_{\mathrm{s}} C_{\mathrm{pl}}}{C_{\mathrm{s}}+C_{\mathrm{pl}}}\right|, \\
C_{\mathrm{pl}} & =\frac{1}{i \omega Z_{\mathrm{pl}}},
\end{aligned}
$$

where $Z_{\mathrm{pl}}$ represents the impedance of the plasma region. Equation (2) indicates that $C_{\mathrm{pr}}$ takes minimum values when $C_{\mathrm{pl}} \rightarrow 0$. This indicates that parallel resonance frequency is independent of existence of the sheath. The probe equivalent capacitances of a short cylindrical probe including and excluding the sheath are calculated as shown in Fig. 2. In the calculations, we used the analytical formula of $Z_{\mathrm{pl}}$ derived by Balmain (1964). It can be seen that $C_{\mathrm{pr}}$ takes a minimum value at the UHR frequency. Another parallel resonance appears at the modi ed plasma resonance (MPR) frequency, which depends on the angle between the probe axis and the static magnetic eld (Ejiri et al., 1968). On the other hand, $C_{\text {pr }}$ shows a peak value not at the cyclotron frequency but at the sheath resonance (SHR) frequency which is higher than the cyclotron frequency (e.g., Oya, 1965; Aso, 1973). It should be noted that the equivalent probe capacitance at the suf ciently lower frequency than the SHR frequency is almost equal to the sheath capacitance $C_{\mathrm{pr}} \approx C_{\mathrm{s}}$.

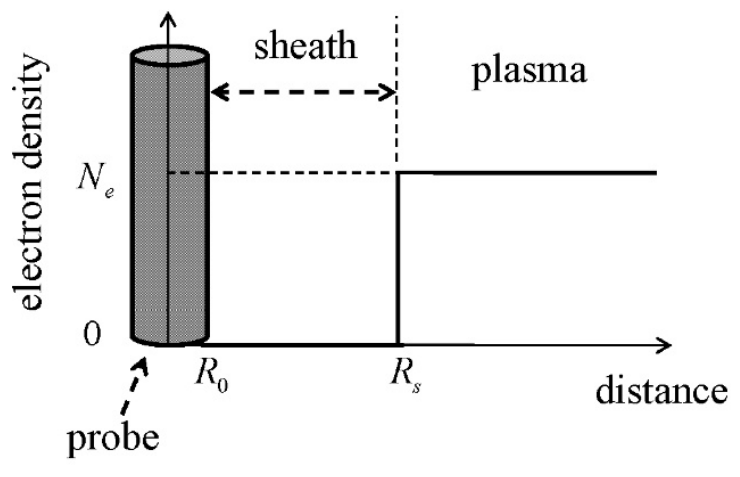

Fig. 1. Applied sheath model. There are no electrons in the sheath region.

The capacitance of a cylindrical sheath is expressed as

$$
C_{\mathrm{s}}=\frac{2 \pi \varepsilon_{0} L}{\ln \left(R_{\mathrm{s}} / R_{0}\right)}
$$

where $L$ and $R_{0}$ are the probe length and the probe radius, respectively. The relation between the sheath radius $R_{\mathrm{s}}$ and the probe potential $\phi_{0}$ can be obtained by solving Poisson's equation (see Jastrow and Pearse, 1957)

$$
-4 \frac{\varepsilon_{0} \phi_{0}}{e N_{\mathrm{e}}}=R_{\mathrm{s}}^{2}\left[\ln \left(\frac{R_{\mathrm{s}}}{R_{0}}\right)^{2}-1\right]+R_{0}^{2} .
$$

Note that we set the space potential as the reference potential in this article.

The remaining issue is how to evaluate the electron temperature $T_{\mathrm{e}}$ from the sheath radius or the probe potential. Some previous works (e.g., Aso, 1973; Steigies et al., 2000; Wakabayashi and Ono, 2006) assumed that the thickness of the sheath is proportional to the Debye length $\lambda_{\mathrm{D}}=\sqrt{\varepsilon_{0} k_{\mathrm{B}} T_{\mathrm{e}} / e^{2} N_{\mathrm{e}}}$ as

$$
R_{\mathrm{s}}-R_{0}=\alpha \lambda_{\mathrm{D}}
$$

However, the value of the coef cient $\alpha$ is arbitrary in the range of about $1-5$. We have therefore evaluated the probe potential by applying the Langmuir probe theory to avoid the uncertainty. Maxwellian distribution yields the electron current $I_{\mathrm{e}}$ as

$$
I_{\mathrm{e}}=e N_{\mathrm{e}} S_{\mathrm{p}} \sqrt{\frac{k_{\mathrm{B}} T_{\mathrm{e}}}{2 \pi m_{\mathrm{e}}}} \exp \left(\frac{e \phi_{0}}{k_{\mathrm{B}} T_{\mathrm{e}}}\right),
$$

where $S_{\mathrm{p}}$ denotes the probe surface area. Since the sheath thickness in the E-F region ionosphere proved to be longer than or comparable to the probe radius, we have adopted the thick sheath approximation to obtain an analytical formula of the ion current $I_{\mathrm{i}}$

$$
\begin{aligned}
I_{\mathrm{i}}= & e N_{\mathrm{e}} S_{\mathrm{p}} \sqrt{\frac{k_{\mathrm{B}} T_{\mathrm{i}}}{2 \pi m_{\mathrm{i}}}}\left[\exp \left(-\frac{e \phi_{0}}{k_{\mathrm{B}} T_{\mathrm{i}}}\right) \operatorname{erfc} \sqrt{-\frac{e \phi_{0}}{k_{\mathrm{B}} T_{\mathrm{i}}}}\right. \\
& \left.+\frac{2}{\sqrt{\pi}} \sqrt{-\frac{e \phi_{0}}{k_{\mathrm{B}} T_{\mathrm{i}}}}\right]
\end{aligned}
$$

where $T_{\mathrm{i}}, m_{\mathrm{i}}$ and $\operatorname{erfc}(x)$ represent the ion temperature, ion mass and the complementary error function, respectively 


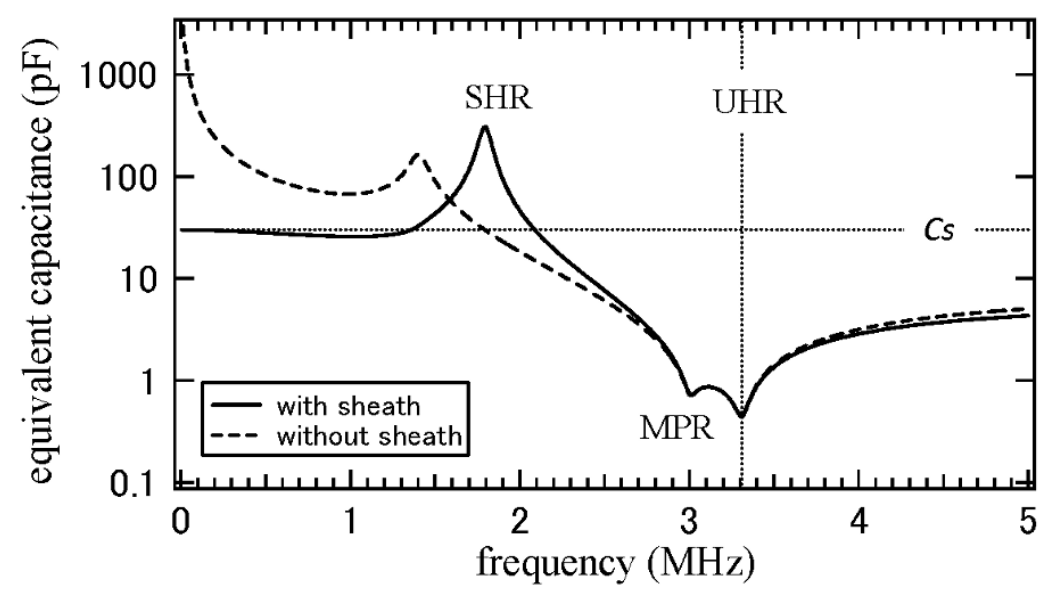

Fig. 2. Calculated equivalent probe capacitance. Plasma parameters are set as $f_{\mathrm{pe}}=3.0 \mathrm{MHz}, f_{\mathrm{ce}}=1.4 \mathrm{MHz}, f_{\mathrm{v}}=0.05 \mathrm{MHz}$, and the static magnetic eld is perpendicular to the probe axis. The solid line represents calculation including the sheath capacitance of $C_{\mathrm{s}}=30 \mathrm{pF}$. Dashed line shows equivalent probe capacitance of only the plasma region.

(Chen, 1965; Maassberg and Isensee, 1981). By solving equation $I_{\mathrm{e}}=I_{\mathrm{i}}$ numerically with the acceptable assumption $T_{\mathrm{i}}=T_{\mathrm{e}}$, the oating potential of a cylindrical probe in a Maxwellian plasma $\phi_{\mathrm{f}}$ can be expressed as the following form:

$$
\phi_{\mathrm{f}}=A_{\mathrm{i}} \frac{k_{\mathrm{B}} T_{\mathrm{e}}}{e},
$$

where $A_{\mathrm{i}}$ is a factor depending on the ion species (e.g., $\left.A_{\mathrm{H}^{+}}=-2.96, A_{\mathrm{O}^{+}}=-4.20, A_{\mathrm{NO}^{+}}=-4.49\right)$. Note that we have already con rmed that thin sheath approximation showed almost the same results for analysis in Section 4, compared with that for the thick sheath approximation.

Combining Eqs. (4), (5), and (9) gives the following relation:

$$
-4 A_{\mathrm{i}} \lambda_{\mathrm{D}}^{2}=\left[R_{0} \exp \left(\frac{2 \pi \varepsilon_{0} L}{C_{\mathrm{s}}}\right)\right]^{2}\left(\frac{4 \pi \varepsilon_{0} L}{C_{\mathrm{s}}}-1\right)+R_{0}^{2} .
$$

This equation explicitly shows that the sheath capacitance $C_{\mathrm{s}}$ gives the Debye length $\lambda_{\mathrm{D}}$. The electron temperature is therefore deduced from the observed sheath capacitance if the applied sheath model is valid.

\section{Outline of the Sounding Rocket Experiments and Instruments}

\subsection{Sounding rocket S-520-23 experiment}

Wind measurement for Ionized and Neutral atmospheric Dynamics study (WIND) campaign was carried out to investigate the momentum transfer between the ionospheric plasma and the neutral atmosphere. The S-520-23 sounding rocket was launched from the Uchinoura Space Center $\left(31.15^{\circ} \mathrm{N}, 131.04^{\circ} \mathrm{E}\right.$ in geodetic coordinates), Japan on 2 nd September 2007, at 19:20 LT $(\mathrm{LT}=\mathrm{UT}+9 \mathrm{~h})$. The payload reached an apex altitude of $279 \mathrm{~km}$ at $268 \mathrm{sec}$ after the launch. The Lithium Ejection System (LES) installed on the S-520-23 rocket was designed to release Li gas three times in the descending phase. The resonantly scattered light of the Li clouds was successfully observed from several ground sites to measure thermospheric neutral wind accurately.
All instruments performed successfully during the rocket ight. Many interesting results were achieved by the neutral wind, the electron density, the electron temperature, the plasma wave, and the electric eld measurements. The present paper focuses on the sheath capacitance measured by the impedance probe instrument.

\subsection{Sounding rocket $\mathbf{S - 3 1 0 - 3 5}$ experiment}

The sounding rocket S-310-35 experiment was performed as a part of the Dynamics and Energetics of the Lower Thermosphere in Aurora (DELTA) campaign, whose objectives and results were described in detail in Abe et al. (2006a) and references therein. The sounding rocket was launched from the Andøya rocket range $\left(69.29^{\circ} \mathrm{N}\right.$, $16.01^{\circ} \mathrm{E}$ in geodetic coordinates) in Norway on 13 th December 2004, at 01:33 LT (LT $=\mathrm{UT}+1 \mathrm{~h})$.

While an auroral breakup was occurring simultaneously with the launch, the sounding rocket ew through the auroral active region. There were two auroral active arcs which crossed the rocket trajectory. The second arc around 124$131 \mathrm{~km}$ altitude was within the range of sheath analysis. We should also mention that observations by the $\mathrm{N}_{2}$ temperature instrument (NTV), which emitted the arti cial electron beam (Kurihara et al., 2006), onboard the rocket affected the electron densities and the probe potentials below about $115 \mathrm{~km}$ altitude in the ascent (see Wakabayashi and Ono, 2006).

\subsection{Impedance probe onboard the sounding rockets}

The impedance probe instrument onboard the S-310-35 sounding rocket was described in Wakabayashi and Ono (2006). A similar impedance probe was installed on the S-520-23 sounding rocket. The capacitance bridge circuit measured the equivalent capacitance of a monopole antenna, whose length and radius were $1.0 \mathrm{~m}$ and $6 \mathrm{~mm}$, respectively. Figure 3 shows the block diagram of the impedance probe instrument called NEI (Number density of Electrons by Impedance probe). The sensors of the impedance probes were connected to the electric ground level with a $20 \mathrm{M} \Omega$ resistor in order to protect the electric circuit from the high energy particles input due to the electron beam injection as well as the auroral particles precip- 


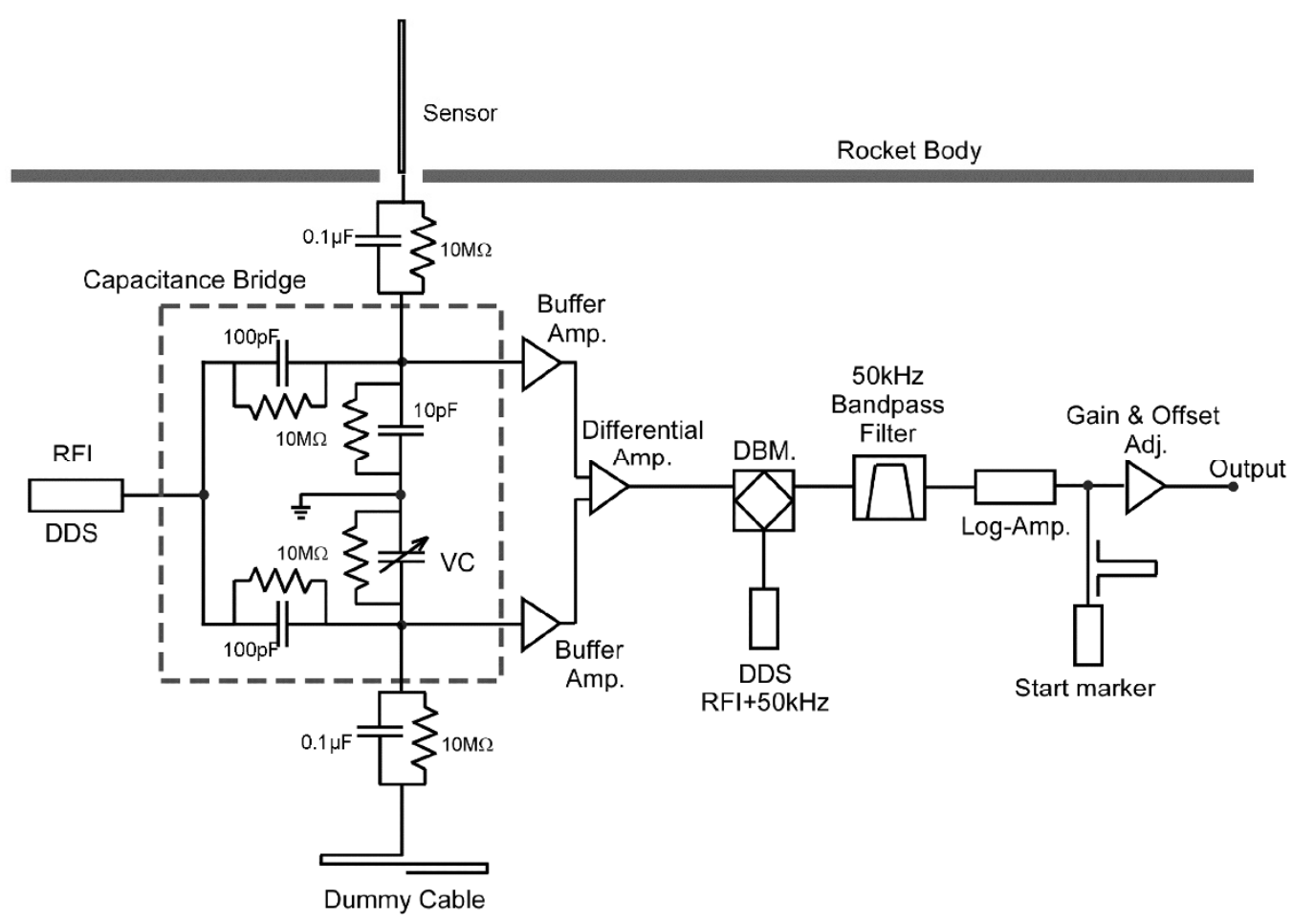

Fig. 3. Block diagram of the impedance probe instrument onboard the sounding rockets S-310-35 and S-520-23.

itation. The capacitance of the variable condenser and the length of the dummy cable were properly adjusted before the ight to eliminate the effects of the stray capacitance.

While other impedance measurement system found that for suf ciently dense plasma, the parallel circuit resonance appeared at less than the UHR frequency due to the stray capacitance (Kiraga, 2003), the NEI systems from the rocket ights S-520-23 and S-310-35 have not observed the parallel circuit resonance. Pre- ight environmental tests performed in the space plasma simulation chamber at ISAS/JAXA also ensured the reproducibility of the results with well de ned, sharp absolute minimum which can be assigned to the upper hybrid frequency. Data pertinent to space ambient plasma show up the same signature of the upper hybrid frequency. The NEI instruments onboard the S-520-23 and S-310-35 rockets therefore realized the accurate measurements of the electron density without the effect of the stray capacitance.

The NEI systems of S-310-35 and S-520-23 are designed to measure the equivalent probe capacitance over the frequency ranges of $300 \mathrm{kHz}-10.3 \mathrm{MHz}$ and $300 \mathrm{kHz}-$ 12.0 MHz, respectively. The frequency resolution of the impedance probe onboard S-310-35 was $10.0 \mathrm{kHz}$ from (0.3-4.3) $\mathrm{MHz}$ and $20 \mathrm{kHz}$ from (4.3-10.3) $\mathrm{MHz}$. In the case of S-520-23, the frequency resolution was $9.4 \mathrm{kHz}$ from (0.3-2.0) $\mathrm{MHz}, 20.0 \mathrm{kHz}$ from (2.0-4.0) $\mathrm{MHz}$, $50.0 \mathrm{kHz}$ from (4.0-8.0) MHz, and $100.0 \mathrm{kHz}$ from $(8.0-$ 12.0) $\mathrm{MHz}$. The time resolution of the impedance probes was about $500 \mathrm{msec}$.

The observed frequency variations of the equivalent probe capacitance provide the sheath capacitances and the UHR frequencies. In the following analysis, we identi ed the equivalent probe capacitance measured at around the
$300 \mathrm{kHz}$, which was suf ciently lower than the observed SHR frequencies, as the sheath capacitance. Electron densities along the rocket trajectory were calculated from the observed UHR frequencies and International Geomagnetic Reference Field (IGRF) model. We also used the electron temperature data measured by the fast Langmuir probes onboard the rockets to obtain the Debye length. The Langmuir probe data were running averaged to reduce the effects of the rocket wake.

\section{Observations of the Sheath Capacitance \\ 4.1 Observations during the ascent of the S-520-23 sounding rocket}

The sensor of the impedance probe was extended at $56.5 \mathrm{sec}$ after the rocket launch, and measurements were successfully operated above $93.1 \mathrm{~km}$. During the ascending phase of the S-520-23 sounding rocket, measurements were performed under quiet plasma conditions. Although the impedance probe was exposed to the sun during the observations, the effects of photoelectrons on the probe potential are negligible in the ionosphere.

Figure 4 shows height variations of the sheath capacitance observed in the ascent of the S-520-23 sounding rocket. The black line represents the sheath capacitance 'measured' at $300 \mathrm{kHz}$. A sheath capacitance curve 'calculated' from the electron densities and the electron temperatures based on the method described in Section 2 is also plotted in the gure. The measured sheath capacitances showed almost the same pro le with that of the calculated curve. However, some differences can be found between the measurements and the calculations below $220 \mathrm{~km}$ altitude. In this altitude range, electron plasma frequencies showed lower values than the electron cyclotron frequen- 


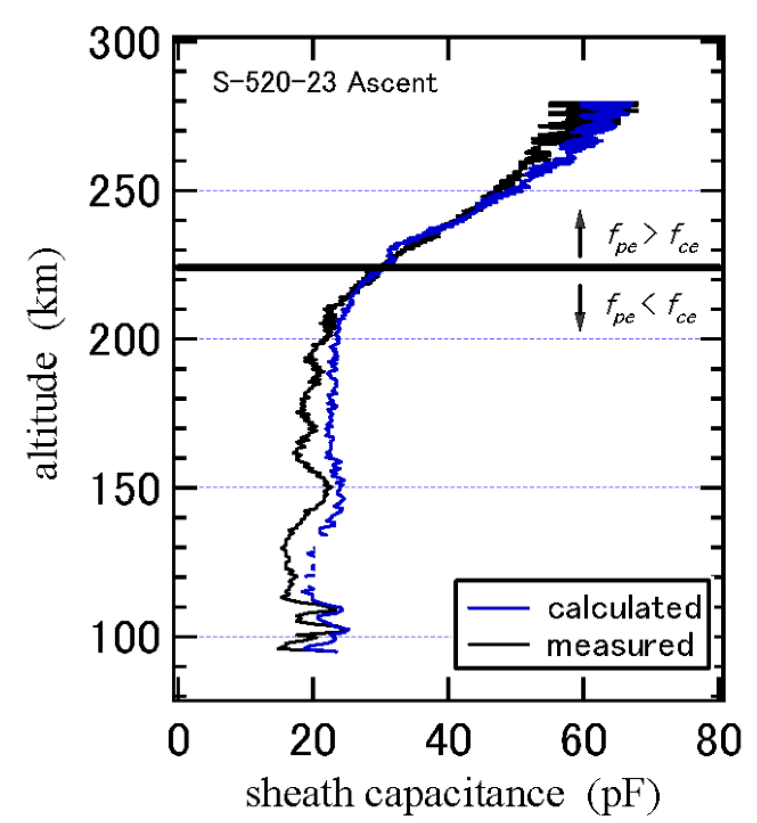

Fig. 4. Measured sheath capacitance in the ascent of the S-520-23 sounding rocket and the sheath capacitance calculated from the Debye length. In the calculation, we used the factor $A_{\mathrm{NO}^{+}}=-4.49$.

cies. The difference and validation of the analysis method are discussed in Section 5.1.

4.2 Observations during the descent of the S-520-23 sounding rocket

In the descent of the S-520-23 sounding rocket, LES released $\mathrm{Li}$ gas. As the Li releases were strongly disturbing the equivalent probe capacitance, it became difficult to deduce the electron densities by detecting the UHR frequencies after the each Li release. On the other hand, the equivalent probe capacitances measured at lower frequency than SHR frequency were not so fluctuated as capacitance curves around the UHR frequency even after the Li releases.

We therefore plotted the equivalent probe capacitance observed at the $300 \mathrm{kHz}$ as a profile of the sheath capacitance (Fig. 5). A notable feature is that measured sheath capacitance indicated sharp enhancements after each Li release. The sheath capacitance observed in the ascending phase showed no such enhancements (see Fig. 4).

The most likely interpretation is that Li release caused increases of the plasma density. The observed sheath capacitance variation corresponded to the increase of the electron density of about 1-2 orders of magnitude. The effects of the chemical release on the ionosphere are intriguing (e.g., Szuszczewicz et al., 1996). Uemoto et al. (2010) includes detailed observations and discussion of the electron density after the Li releases.

Here, we should emphasize the practicality of the plasma diagnosis from the sheath capacitance. In rocket observations, instruments sometimes fail to measure plasma parameters due to artificial interferences. Analysis of the sheath capacitance in addition to the UHR frequency will contribute to improvement of the success rate of plasma measurements.

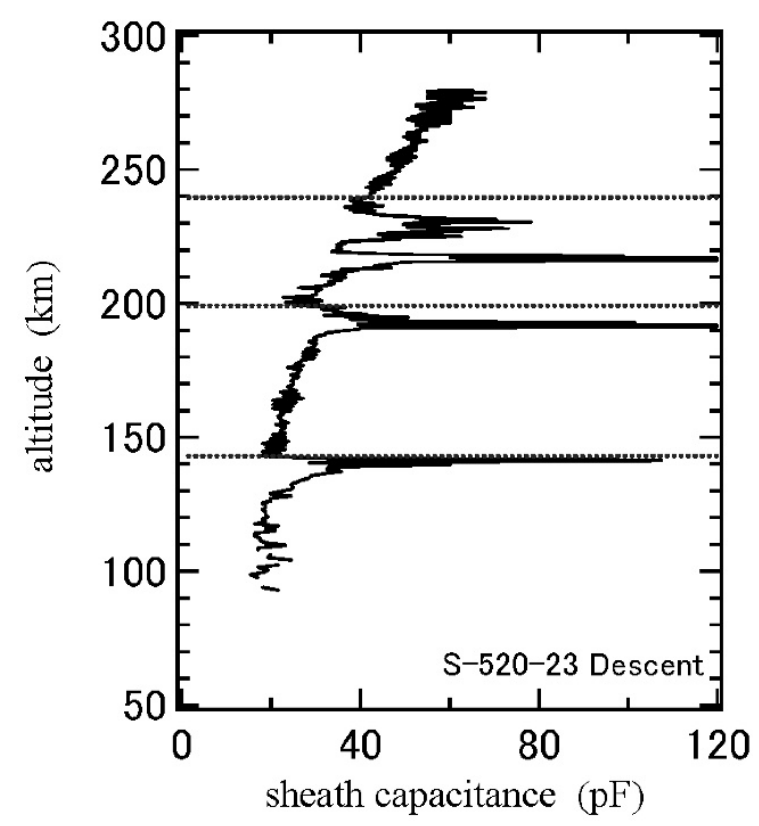

Fig. 5. Sheath capacitance observed during the descent of the S-520-23 sounding rocket. Dashed lines represent the planned altitudes to start $\mathrm{Li}$ release.

\subsection{Observations in the auroral ionosphere}

We have also examined the sheath capacitance observed by the impedance probe onboard the S-310-35 sounding rocket, which was launched into the auroral ionosphere. The electron density and the electron temperature measurements along the rocket trajectory were reported in detail by Wakabayashi and Ono (2006) and Abe et al. (2006b), respectively. Figure 6 summarizes the analysis of the sheath capacitance in the case of the S-310-35 experiment. It can be clearly seen that the measured sheath capacitances showed lower values than the expected ones. The difference between the measurements and calculations was significantly large in contrast with the results of the S-52023 ascent. The large fluctuation of the sheath capacitance observed below $115 \mathrm{~km}$ altitude in the ascent was caused by the potential change according to the artificial electron beam emitted by the NTV instrument. Periodic modulation of the sheath capacitance, which was remarkable during the descent observation, came from the rocket wake formed on the opposite side of the moving direction.

\section{Discussion}

\subsection{Validation of the calculation method}

The first point that should be discussed is the validity of the evaluation method of the sheath capacitance. Figure 7 shows the difference between the measured sheath capacitance and the calculated one in the ascending phase of the S-520-23 sounding rocket. Figure 7(b) shows that most of the sheath capacitances, which were observed in the condition $f_{\mathrm{pe}}>f_{\mathrm{ce}}$, were within $\pm 10 \%$ different from that of the calculations. The difference is found to be independent from the plasma frequency. It follows that the sheath capacitance, which can be obtained as the probe capacitance at a sufficiently lower frequency than the SHR frequency, allows us to estimate the Debye length. From another point 

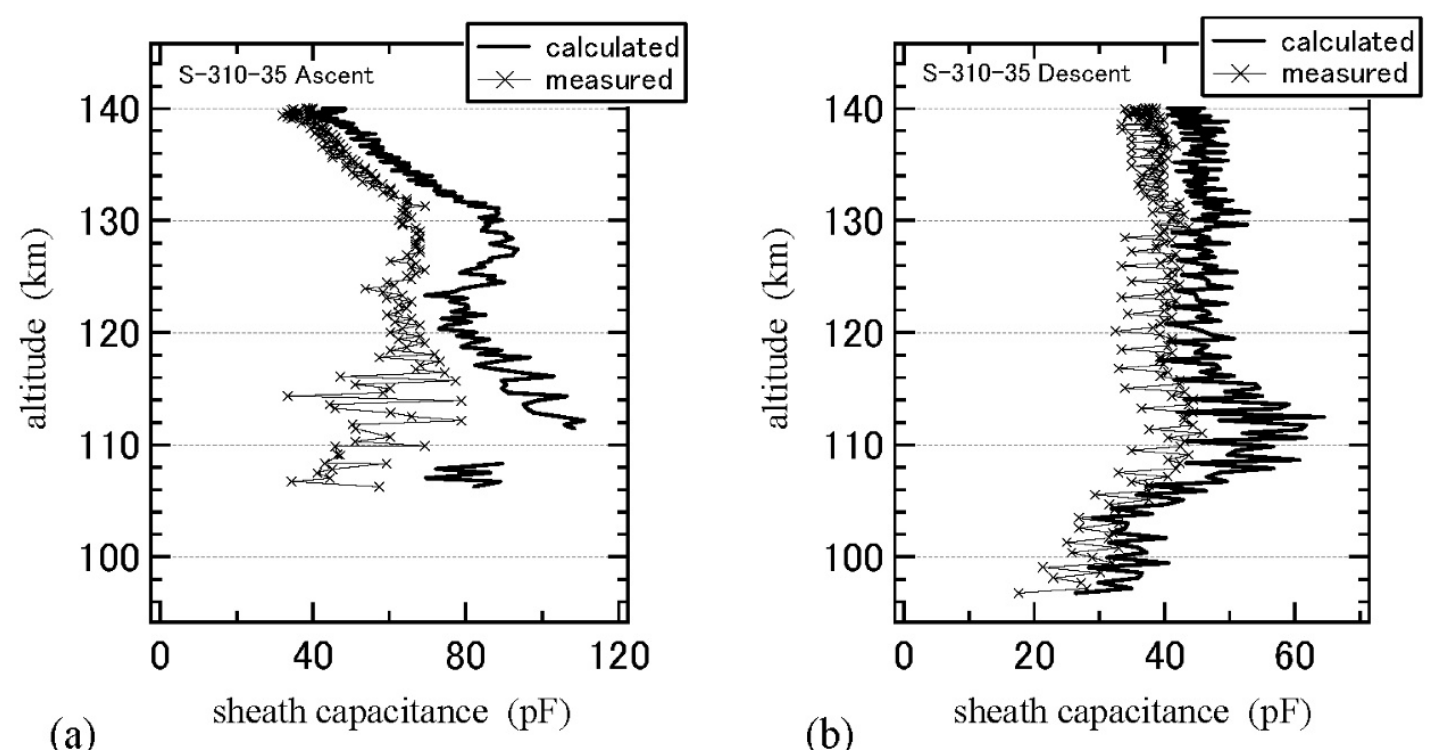

Fig. 6. Measured sheath capacitance in the S-310-35 experiment and calculated sheath capacitance.

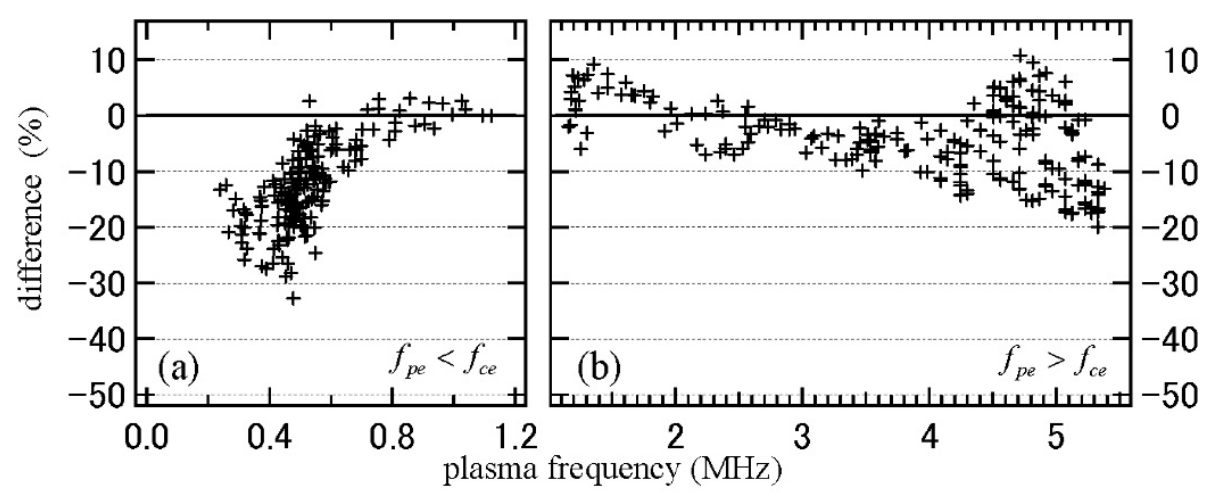

Fig. 7. Difference between the observed sheath capacitance and the calculated ones in the ascent of the S-520-23 rocket. The difference is de ned as $\left(C_{\text {s_measured }}-C_{\text {s_calculated }}\right) / C_{\text {_calculated }} \times 100$.

of view, certainty of the electron density and electron temperature measurements was con rmed by the analysis.

In contrast, observations with $f_{\mathrm{pe}}<f_{\text {ce }}$ seemed to make the difference large (Fig. 7(a)). It can be seen that the measured sheath capacitance showed about $10-30 \%$ lower values compared with the calculated values, even though the ionospheric plasma was not disturbed. Where does the difference come from? The measurement examples of the equivalent probe capacitance are shown in Fig. 8. The dashed line denotes the sheath capacitance calculated from the observed Debye length. When $f_{\mathrm{pe}}>f_{\text {ce }}$ was satis ed, the probe capacitance curve approached the calculated capacitance at low frequency (Fig. 8(a)). This demonstrated that the equivalent probe capacitance observed at $300 \mathrm{kHz}$ can be recognized as the sheath capacitance. On the other hand, the equivalent probe capacitance at a frequency lower than the SHR frequency was not at (Fig. 8(b)). There was an additional dip of the equivalent probe capacitance at $0.57 \mathrm{MHz}$, which corresponded to the plasma frequency. The dip was thus identi ed as a plasma resonance, which was not clear with a condition of $f_{\mathrm{pe}}>f_{\text {ce }}$. Due to the existence of the plasma resonance, the equivalent probe capacitances of around the $300 \mathrm{kHz}$ re ected the plasma impedance as well as the sheath capacitance. We concluded that a condition $f_{\text {pe }}>f_{\text {ce }}$ is suitable for accurate plasma diagnostics via the method described in this paper.

We also found a slight difference of the sheath capacitance in the plasma frequency range of more than $4.5 \mathrm{MHz}$, even though $f_{\text {pe }}>f_{\text {ce }}$ was satis ed (see Fig. 7(b)). The difference appeared periodically around the apex of the rocket ight. The periodic variation was caused by the rocket spin. The effect of rocket wake became signi cant near the apex and descending phase due to the rocket attitude. Since the electron temperature data were running averaged, the calculated sheath capacitances do not re ect the electron temperatures in the wake. There are some physical issues regarding how to evaluate the temperature in the wake. It is beyond the scope of this article to examine the sheath capacitance measured in the wake region.

\subsection{Effect of the auroral particles precipitation}

As shown in Fig. 6, the sheath capacitances measured by the S-310-35 rocket were signi cantly lower than that of the calculations. Note that the plasma frequencies were higher than the cyclotron frequencies except for the altitudes below 

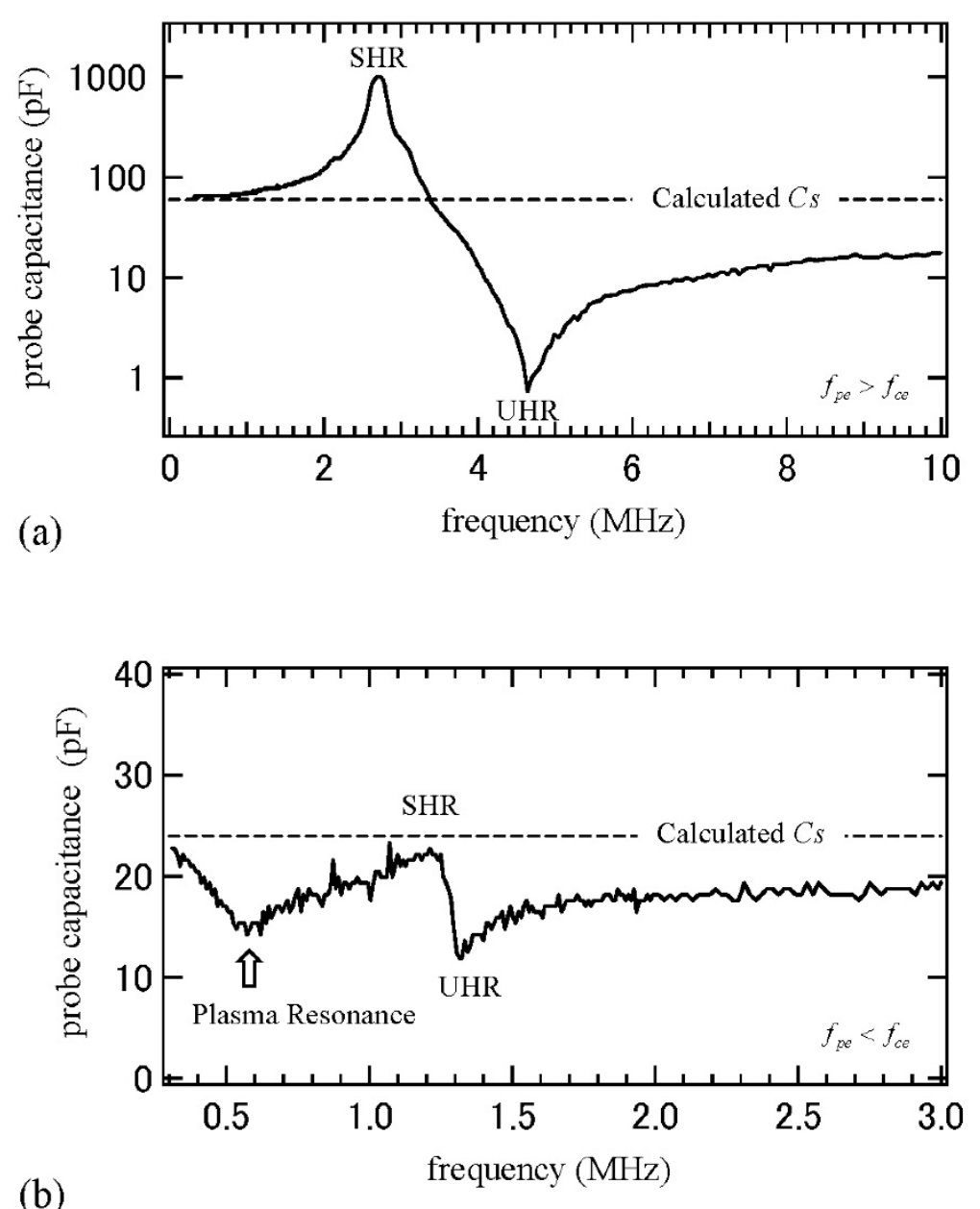

Fig. 8. Observed spectra of the equivalent probe capacitance. (a) Measurement at $279.3 \mathrm{~km}$ altitude in a condition of $f_{\mathrm{pe}}=4.51 \mathrm{MHz}$ and $f_{\mathrm{ce}}=1.11 \mathrm{MHz}$, (b) measurement at $150.2 \mathrm{~km}$ altitude in a condition of $f_{\mathrm{pe}}=0.58 \mathrm{MHz}$ and $f_{\mathrm{ce}}=1.20 \mathrm{MHz}$.

$100 \mathrm{~km}$, due to the ionization by auroral particles precipitation (Wakabayashi and Ono, 2006). This indicates that the difference between the observations and calculations was not derived from the condition $f_{\mathrm{pe}}<f_{\text {ce }}$ as discussed in Section 5.1.

The difference should be caused by a charge-up effect of the electrode due to the auroral particles (Watanabe, 2000). While the probe potential $\phi_{0}$ in a Maxwellian plasma is equal to $\phi_{\mathrm{f}}$, the probe potential is predicted to be negatively biased $\left(\phi_{0}<\phi_{\mathrm{f}}\right)$ in the auroral ionosphere due to the non-Maxwellian distribution. We therefore recalculated the sheath capacitance with the probe capacitance treated as a free parameter. Consequently, we found that the observed sheath capacitances were well tted to the calculated sheath capacitances with the probe potential $\phi_{0}=2 \phi_{\mathrm{f}}$ as shown in Fig. 9. The estimated probe potential was from -1.2 to -0.2 volts, which was the same order as that with a rocket potential observation by the fast Langmuir probe as reported in Abe et al. (2006b).

Figure 10 enlarges a portion of the Fig. 9(a). The shaded region in the gure shows an altitude range where strong particle precipitation was observed by the Auroral Particle Detector (APD) instrument (Ogasawara et al., 2006). In the region, measured sheath capacitances indicated lower values than the calculated ones with the probe potential $\phi_{0}=2 \phi_{\mathrm{f}}$. The probe potential of about $\phi_{0}=2.5-3.0 \phi_{\mathrm{f}}$ was found to be necessary to explain the observed sheath capacitances. Therefore, we concluded that the sheath capacitance re ected the intensity of auroral particle precipitation.

\section{Conclusions}

We examined the sheath capacitance measured by using impedance probe techniques. The sheath capacitance was analytically calculated from the Debye length. Such calculations are a simpler method in comparison with the PIC simulations (Miyake et al., 2008), and as a result, our method does not then require enormous computer facility.

We have compared the sheath capacitances observed by the impedance probes onboard the sounding rockets with the calculated sheath capacitances. When a condition of $f_{\text {pe }}>f_{\text {ce }}$ was satis ed, the equivalent probe capacitance observed at a suf ciently lower frequency than the SHR frequency showed a constant value which equaled to the sheath capacitance. The observed sheath capacitances agreed well with the calculations. This indicates that the calculation methodology of the sheath capacitance described in Section 2 is valid; and the Debye length can be estimated from the observed sheath capacitance. The advantages of this technique are summarized as follows: (a) the sheath capaci- 

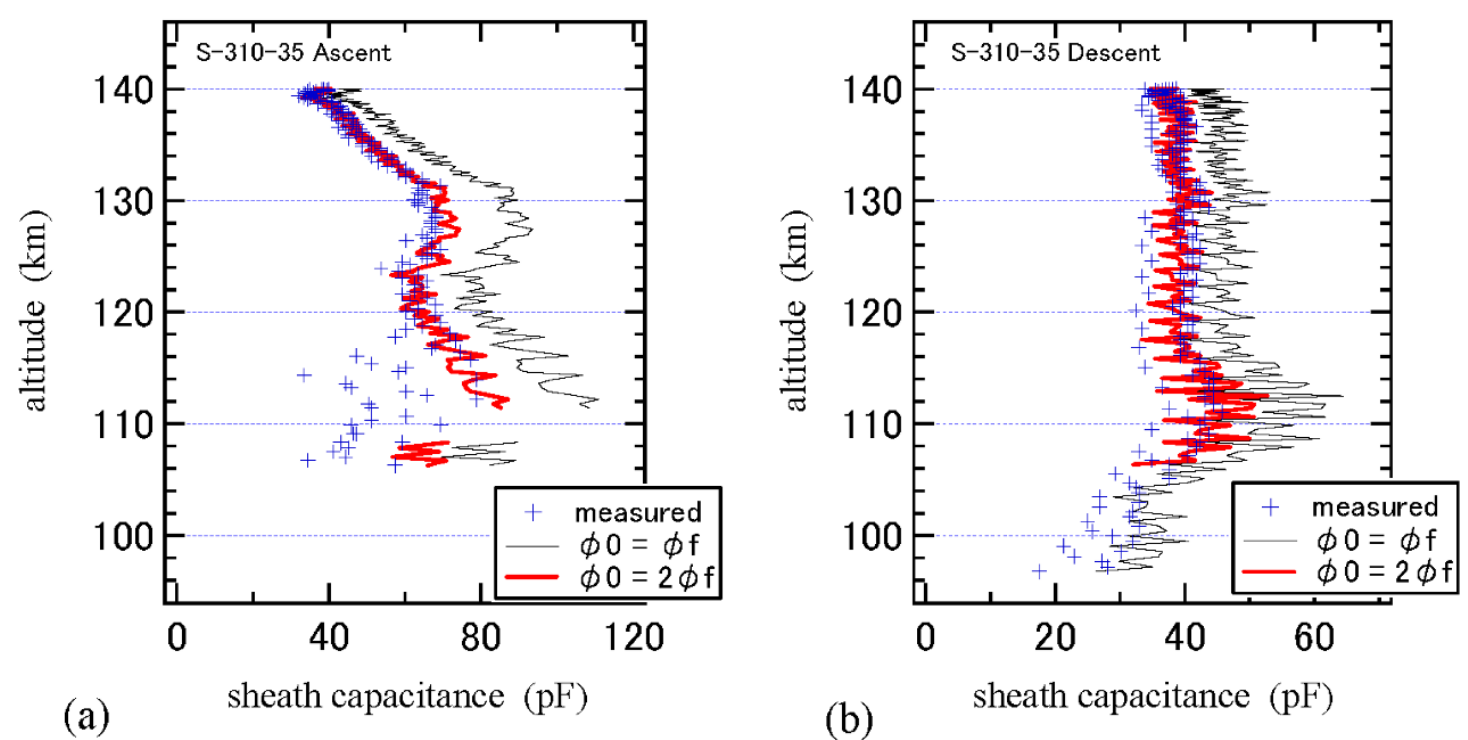

Fig. 9. Measured sheath capacitance in the S-310-35 experiment and calculated sheath capacitance. The red line denotes the calculated sheath capacitance with the probe potential of $\phi_{0}=2 \phi_{\mathrm{f}}$.

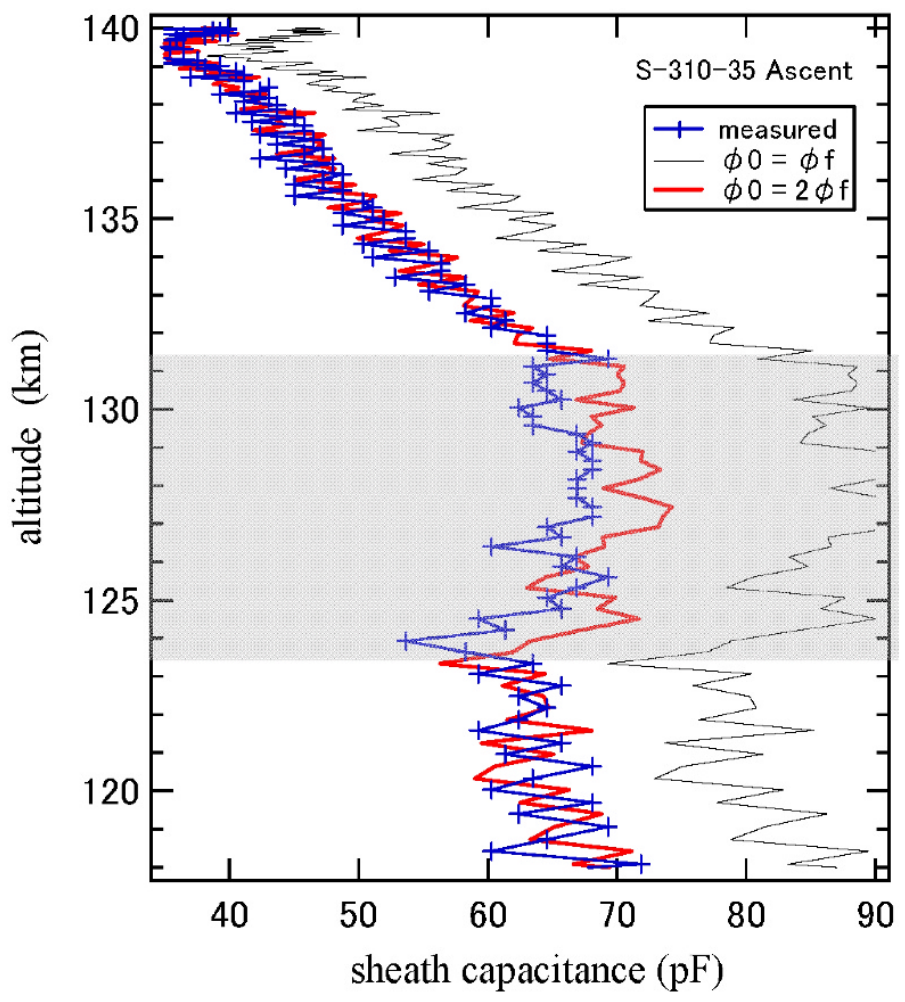

Fig. 10. Extended view of Fig. 9(a). The shaded range shows the region where the auroral arc appeared.

tance in addition to the UHR frequency provides plasma parameters, (b) the electron density, the electron temperature and the probe potential measured via other instruments can be cross-checked with analysis of the sheath capacitance, and (c) the analysis method is simple. Since the detection of the UHR frequencies sometimes becomes hard due to plasma disturbances (e.g., effect of Li release and electron beam emission) in sounding rocket experiments, plasma diagnosis technique from the sheath capacitance is valuable. In future experiments, we propose to design the impedance probe to measure the sheath capacitance with high time resolution. This improvement allows the impedance probe to realize both high-accurate measurements of the electron density from the UHR frequency and high-resolution plasma diagnostics from the sheath capacitance.

In the case of $f_{\mathrm{pe}}<f_{\mathrm{ce}}$, the plasma resonance clearly appeared. As a result, it became difficult to separate the sheath capacitance from the observed the equivalent probe capacitance. For accurate plasma diagnostics from the sheath capacitance obtained by the impedance probe, it is necessary 
that the plasma frequency is larger than the cyclotron frequency.

Observations in the auroral ionosphere indicated that the probe potential was shifted from the analytical solution of the oating potential due to the non-Maxwellian velocity distribution. Strong precipitation of energetic electrons in the auroral arc should have caused the lower probe potential. Quantitative discussion of the response of the probe potential to the total ux of precipitating auroral particles remains a future issue to examine.

Acknowledgments. The sounding rocket experiments were conducted by the Institute of Space and Astronautical Science, Japan Aerospace Exploration Agency (ISAS/JAXA) as international projects. We thank all members of the rocket experiments. Preight operation tests of the impedance probes were supported by the Space Plasma Laboratory, ISAS/JAXA. The impedance probes were manufactured by System Keisoku Co., Ltd. This work is supported by the Global COE Program "Global Education and Research Center for Earth and Planetary Dynamics" at Tohoku University.

\section{References}

Abe, T., J. Kurihara, N. Iwagami, S. Nozawa, Y. Ogawa, R. Fujii, H. Hayakawa, and K. I. Oyama, Dynamics and Energetics of the Lower Thermosphere in Aurora (DELTA)_Japanese sounding rocket campaign—, Earth Planets Space, 58, 1165-1171, 2006a.

Abe, T., K. I. Oyama, and A. Kadohata, Electron temperature variation associated with the auroral energy input during the DELTA campaign, Earth Planets Space, 58, 1139-1146, 2006b.

Aso, T., A sheath resonance observed by a high frequency impedance probe, J. Geomag. Geoelectr., 25, 325-330, 1973.

Balmain, K. G., The impedance of a short dipole antenna in a magnetoplasma, IEEE Trans. Antennas Propag., AP-12, 605-617, 1964.

Barjatya, A. and C. M. Swenson, Observation of triboelectric charging effects on Langmuir-type probes in dusty plasma, J. Geophys. Res., 111, A10302, 2006.

Béghin, C., P. M. E. Décréau, J. Pickett, D. Sundkvist, and B. Lefebvre, Modeling of Cluster's electric antenna in space: Application to plasma diagnostics, Radio Sci., 40, RS6008, 2005.

Blackwell, D. D., D. N. Walker, S. J. Messer, and W. E. Amatucci, Antenna impedance measurements in a magnetized plasma. II. Dipole antenna, Phys. Plasmas, 14, 092106, 2007.

Chen, F. F., Electric probes, in Plasma Diagnostic Techniques, edited by R. H. Huddlestone and S. L. Leonard, 113 pp, Academic Press, New York and London, 1965.

Chen, F. F., Time-varying impedance of the sheath on a probe in an RF plasma, Plasma Sources Sci. Technol., 15, 773-782, 2006.

Ejiri, M., H. Oya, and T. Obayashi, A modi ed plasma resonance observed by a rocket-borne gyro-plasma probe, Rep. Ionos. Space Res. Jpn., 22, 201-204, 1968.

Ejiri, M., H. Oya, T. Aso, K. Morita, T. Obayashi, S. Urimoto, and H. Yamaki, The gyro-plasma probe onboard the REXS-DENPA satellite, Inst. Space Aeronaut. Sci., Univ. Tokyo, 495, 83-198, 1973.

Jackson, J. E. and J. A. Kane, Measurement of ionospheric electron densities using an RF probe technique, J. Geophys. Res., 64, 1074-1075, 1959.

Jastrow, R. and C. A. Pearse, Atmospheric drag on the satellite, J. Geophys. Res., 62, 413-423, 1957.

Kiraga, A., Equivalent circuit simulation of cylindrical monopole impedance measurements in ionospheric electron plasma, Adv. Space Res., 32, 2355-2360, 2003.

Kurihara, J., T. Abe, K.-I. Oyama, E. Grif n, M. Kosch, A. Aruliah, K. Kauristie, Y. Ogawa, S. Komada, and N. Iwagami, Observations of the lower thermospheric neutral temperature and density in the DELTA campaign, Earth Planets Space, 58, 1123-1130, 2006.

Maassberg, H. and U. Isensee, Symmetric theory of probe-plasma interactions, Planet. Space. Sci., 29, 555-577, 1981.
Miyake, Y., H. Usui, H. Kojima, Y. Omura, and H. Matsumoto, Electromagnetic Particle-In-Cell simulation on the impedance of a dipole antenna surrounded by an ion sheath, Radio Sci., 43, RS3004, 2008.

Ogasawara, K., K. Asamura, T. Takashima, Y. Saito, and T. Mukai, Rocket observation of energetic electrons in the low-altitude auroral ionosphere during the DELTA campaign, Earth Planets Space, 58, 1155-1164, 2006.

Oya, H., Effect of resonances on the admittance of an RF plasma probe surrounded by an ion sheath, Rep. Ionos. Space Res. Jpn., 19, 243-271, 1965.

Oya, H. and T. Aso, Ionospheric electron temperature measured by a gyroplasma probe, Space Research IX-North-Holland Publishing Comp., 287-296, 1969.

Oya, H. and A. Morioka, Instrumentation and observations of gyro-plasma probe installed on TAIYO for measurement of ionospheric plasma parameters and low energetic particle effects, J. Geomag. Geoelectr., 27, 331-361, 1975.

Oya, H. and T. Obayashi, Measurement of ionospheric electron density by a gyro-plasma probe: A rocket experiment by a new impedance probe, Rep. Ionos. Space Res. Jpn., 20, 199-213, 1966.

Spencer, E., S. Patra, T. Andriyas, C. Swenson, J. Ward, and A. Barjatya, Electron density and electron neutral collision frequency in the ionosphere using plasma impedance probe measurements, J. Geophys. Res., 113, A09305, 2008.

Steigies, C. T., D. Block, M. Hirt, B. Hipp, A. Piel, and J. Grygorczuk, Development of a fast impedance probe for absolute electron density measurements in the ionosphere, J. Phys. D: Appl. Phys., 33, 405-413, 2000.

Suzuki, T., T. Ono, M. Iizima, M. Wakabayashi, and A. Kumamoto, Characteristics of the cyclotron harmonic resonances found by impedance probe experiments in a laboratory plasma, J. Plasma Fusion Res. Ser., 8, 165-168, 2009.

Szuszczewicz, E. P., G. Earle, T. Bateman, Z. Klos, A. Kiraga, and R. W. Schunk, An "in situ" investigation of early time multi-ion expansion processes in an F region chemical release, J. Geophys. Res., 101, 1574915764, 1996.

Takahashi, T., H. Oya, S. Watanabe, and Y. Watanabe, Observation of electron density by the impedance probe on board the Ohzora (EXOSC) satellite, J. Geomag. Geoelectr., 37, 389-411, 1985.

Tsutsui, M., I. Nagano, H. Kojima, K. Hashimoto, H. Matsumoto, S. Yagitani, and T. Okada, Measurements and analysis of antenna impedance aboard the Geotail spacecraft, Radio Sci., 32, 1101-1126, 1997.

Uemoto, J., T. Ono, T. Yamada, T. Suzuki, M.-Y. Yamamoto, S. Watanabe, A. Kumamoto, and M. Iizima, Impact of lithium releases on ionospheric electron density observed by impedance probe during WIND campaign, Earth Planets Space, 62, this issue, 589-597, 2010.

Wakabayashi, M. and T. Ono, Electron density measurement under the in uence of auroral precipitation and electron beam injection during the DELTA campaign, Earth Planets Space, 58, 1147-1154, 2006.

Wakabayashi, M., T. Ono, H. Mori, and P. A. Bernhardt, Electron density and plasma waves in mid-latitude sporadic-E layer observed during the SEEK-2 campaign, Ann. Geophys., 23, 2335-2345, 2005.

Ward, J., C. Swenson, and C. Furse, The impedance of a short dipole antenna in a magnetized plasma via a nite difference time domain model, IEEE Trans. Antennas Propag., 53, 2711-2718, 2005.

Watanabe, S. and H. Oya, Occurrence characteristics of low latitude ionosphere irregularities observed by impedance probe on board the Hinotori satellite, J. Geomag. Geoelectr., 38, 125-149, 1986.

Watanabe, Y., Charging of the impedance-probe by the auroral energetic electrons, Adv. Polar Upper Atmos. Res., 14, 172-178, 2000.

Yamamoto, M.-Y., T. Ono, H. Oya, R. T. Tsunoda, M. F. Larsen, S. Fukao, and M. Yamamoto, Structures in sporadic-E observed with an impedance probe during the SEEK campaign: Comparisons with neutral-wind and radar-echo observations, Geophys. Res. Lett., 25, 1781-1784, 1998.

T. Suzuki (e-mail: t-suzuki@stpp1.geophys.tohoku.ac.jp), T. Ono, J. Uemoto, M. Wakabayashi, T. Abe, A. Kumamoto, and M. Iizima 